\title{
Orientações de enfermagem a pacientes ostomizados: Revisão integrativa
}

\author{
Nursing guidelines for ostomized patients: Integrative review \\ Guías de enfermería para pacientes ostomizados: Revisión integradora
}

Recebido: 10/07/2021 | Revisado: 17/07/2021 | Aceito: 19/07/2021 | Publicado: 26/07/2021

\author{
Juliana Alves Couto \\ ORCID: https://orcid.org/0000-0002-9964-6172 \\ Faculdade de Desenvolvimento do Rio Grande do Sul, Brasil \\ E-mail: ju.alves.couto@ hotmail.com \\ Taiane da Silva de Sá \\ ORCID: https://orcid.org/0000-0002-2594-2534 \\ Faculdade de Desenvolvimento do Rio Grande do Sul, Brasil \\ E-mail: taianeee@hotmail.com \\ Kelly de Souza da Silva \\ ORCID: https://orcid.org/0000-0002-1578-2688 \\ Faculdade de Desenvolvimento do Rio Grande do Sul, Brasil \\ E-mail: kelly.silva@fadergs.edu.br \\ Maurício Rouvel Nunes \\ ORCID: https://orcid.org/0000-0002-4975-6568 \\ Faculdade de Desenvolvimento do Rio Grande do Sul, Brasil \\ E-mail: mrouvelnunes@gmail.com
}

\begin{abstract}
Resumo
Introdução: $\mathrm{O}$ paciente recém estomizado encontra muitas dificuldades para se adaptar a sua nova forma corporal e também apresentam problemas de convívio social. A atuação do enfermeiro como educador em saúde aos pacientes, familiares e cuidadores é uma competência inerente à prática assistencial. Objetivo: Descrever as evidências científicas disponíveis na literatura sobre as orientações de enfermagem a pacientes estomizados. Métodos: Trata-se de uma revisão integrativa da literatura, no período de 2016 a 2021, nas bases de dados Lilacs e BDENF. Foram utilizados os seguintes descritores nas bases: "Estomia", "Enfermagem", "Educação em Saúde". Para o cruzamento dos descritores, utilizou-se um protocolo com o booleano AND. Como critérios de inclusão foram selecionados artigos disponíveis nas bases de dados e com texto completo e de livre acesso e estudos disponíveis em português. Os critérios de exclusão foram editoriais de revistas, cartas ao editor e estudos que não abordavam a temática do trabalho. Resultados: Após filtragem com base no fluxograma definido, foram selecionados nove artigos conforme critérios estabelecidos. Após a leitura e análise dos artigos, emergiram três categorias temáticas, os quais foram: "Educação em saúde e a atuação do enfermeiro frente ao paciente ostomizado", "Abordagens de educação em saúde para promover o autocuidado e a reabilitação do paciente estomizado" e "Estratégias e tecnologias para educação permanente das equipes sobre ostomias". Conclusão: conclui-se que as evidências sobre as orientações de pacientes ostomizados são escassas. A educação em saúde de profissionais dos profissionais de enfermagem é de fundamental importância para a promoção do autocuidado dos portadores de estomias.
\end{abstract}

Palavras-chave: Estomia; Cuidados de enfermagem; Educação em saúde.

\begin{abstract}
Introduction: Newly ostomized patients find it very difficult to adapt to their new body shape and also present social problems. The role of the nurse as a health educator for patients, family members and caregivers is an inherent competence in care practice. Objective: To describe the scientific evidence available in the literature on nursing guidelines for ostomy patients. Methods: This is an integrative literature review, from 2016 to 2021, in the Lilacs and BDENF databases. The following descriptors were used in the bases: "Ostomy", "Nursing", "Health Education". For the crossing of descriptors, a protocol with Boolean AND is used. As inclusion criteria, articles available in the databases and with full text and free access and studies available in Portuguese were selected. Exclusion criteria were editorials from journals, letters to the editor and studies that did not address the theme of the work. Results: After filtering based on the defined flowchart, nine articles were selected according to established criteria. After reading and analyzing the articles, three thematic categories emerged, which were: "Health education and the role of nurses facing the ostomy patient", "Health education approaches to promote self-care and rehabilitation of the ostomy patient" and "Strategies and technologies for permanent education of teams about ostomies". Conclusion: it is concluded that the health education of professional nursing professionals is of fundamental importance for the promotion of self-care for ostomy patients.
\end{abstract}

Keywords: Ostomy; Nursing care; Health education. 


\begin{abstract}
Resumen
Introducción: A los pacientes recién sometidos a ostomía les resulta muy difícil adaptarse a su nueva forma corporal y también presentan problemas sociales. El papel de la enfermera como educadora sanitaria para pacientes, familiares y cuidadores es una competencia inherente a la práctica asistencial. Objetivo: Describir la evidencia científica disponible en la literatura sobre guías de enfermería para pacientes ostomizados. Métodos: Se trata de una revisión de literatura integradora, de 2016 a 2021, en las bases de datos Lilacs y BDENF. En las bases se utilizaron los siguientes descriptores: "Ostomía", "Enfermería", "Educación en Salud". Para el cruce de descriptores se utilizó un protocolo con AND booleano. Como criterios de inclusión se seleccionaron los artículos disponibles en las bases de datos y con texto completo y libre acceso y estudios disponibles en portugués. Los criterios de exclusión fueron editoriales de revistas, cartas al editor y estudios que no abordaran el tema del trabajo. Resultados: Después de filtrar en base al diagrama de flujo definido, se seleccionaron nueve artículos según los criterios establecidos. Después de la lectura y análisis de los artículos, surgieron tres categorías temáticas, que fueron: "La educación para la salud y el papel del enfermero ante el paciente ostomizado", "Enfoques de educación sanitaria para promover el autocuidado y la rehabilitación del paciente ostomizado" y "Estrategias y tecnologías para la formación permanente de los equipos sobre ostomías ". Conclusión: se concluye que la educación en salud de los profesionales de enfermería es de fundamental importancia para la promoción del autocuidado de los pacientes ostomizados.
\end{abstract}

Palabras clave: Estomía; Atención de enfermería; Educación en salud.

\title{
1. Introdução
}

Segundo dados da International Ostomy Association (IOA), estima-se que no Brasil, no ano de 2018, existiam aproximadamente 207 mil estomizados (Brasil, 2019). O estoma também chamado de ostoma intestinal de eliminação é um orifício, localizado no abdome, realizado através de um procedimento cirúrgico que constrói uma comunicação de uma parte do intestino com a superfície do corpo que permite que o indivíduo realize suas eliminações de fezes e flatos, que serão depositadas em uma bolsa coletora (Caetano et al., 2013; Andrade et al., 2017). Dependendo da parte do intestino envolvida na confecção do estoma ele pode ser classificado em ileostomia, quando é realizado a partir do íleo, ou colostomia, quando é realizado a partir do cólon. Um estoma pode ser temporário ou definitivo de acordo com a necessidade terapêutica do indivíduo, as causas mais comuns são câncer colorretal, a doença inflamatória intestinal (como retocolite ulcerativa e a doença de Crohn) a polipose adenomatosa familiar entre outras (Silva et al., 2017; Dos Santo et al., 2020). Os pacientes recém estomizados encontram muitas dificuldades para se adaptar a sua nova forma corporal, inicialmente desenvolvem reações emocionais como negação, ira e depressão, além disso, também apresentam problemas de convívio social. Com as modificações fisiológicas advém as necessidades dos cuidados com a bolsa de colostomia, pois com ela surgem incômodos como por exemplo, as eliminações de gases, vazamentos e o odor exalado pela bolsa (Caetano et al., 2013). Na alta hospitalar dos pacientes recém ostomizados, faz-se necessário uma atuação da equipe assistencial no que concerne a melhorar a qualidade de vida desses pacientes, pois as orientações da equipe de enfermagem serão fundamentais para que se possa ter acesso aos serviços da atenção primária em saúde e que se possa manter um acompanhamento das alterações clínicas desses indivíduos (Silva; Shimizu, 2006). A assistência a esses pacientes é um desafio, pois a educação em saúde é uma importante tecnologia de cuidado utilizada pelo profissional enfermeiro para que o indivíduo conviva com sua ostomia (Silva; Shimizu, 2006; De morais et al., 2018). A atuação do enfermeiro como educador em saúde aos pacientes, familiares e cuidadores é uma competência inerente à prática assistencial do enfermeiro, competência que é exercida pelo profissional nos diferentes cenários da prática assistencial, com essa vivência a enfermagem acaba desenvolvendo habilidades ao abordar esse paciente adaptando a passagem das orientações às suas particularidades e limitações a fim de garantir que o aprendizado aconteça de forma efetiva, pois nesse diálogo são passadas informações importantes como higiene e conforto, troca de bolsa e cuidados com a pele periostoma que são de extrema importância para a reabilitação dos pacientes e assim garantir o seu bem estar no pós-alta (Martins; Alvim, 2011). Diante disso, o objetivo do trabalho é descrever as evidências científicas disponíveis na literatura sobre as orientações de enfermagem a pacientes estomizados. 


\section{Metodologia}

Trata-se de uma revisão integrativa que seguiu as seguintes etapas: identificação do tema e questão de pesquisa, estabelecimento dos critérios de inclusão e exclusão dos estudos, categorização dos estudos, avaliação dos estudos incluídos, interpretação dos resultados e apresentação da síntese do conhecimento (Mendes; Silveira; Galvão, 2008). Atendendo ao objetivo anteriormente descrito, definimos a seguinte questão de pesquisa: “Quais são as evidências científicas sobre as orientações de enfermagem a pacientes estomizados?” A pesquisa foi realizada de forma independente por duas pesquisadoras, entre os meses de março e junho de 2021 nas bases de dados: Literatura Latino-Americana e do Caribe em Ciências da Saúde (Lilacs) e Base de Dados em Enfermagem (BDENF). As estratégias de pesquisas aplicadas nas bases de dados foram: (“Estomia”) AND (“Enfermagem”) AND (“Educação em Saúde”). Foram definidos como critério de inclusão: estudos disponíveis nas bases de dados e com texto completo e de livre acesso; estudos disponíveis em português publicados no período de 2016 a 2021. Foram excluídos editoriais de revistas, cartas ao editor e estudos que não abordavam a temática do trabalho. O processo de seleção dos artigos, deu-se em duas etapas: a primeira etapa consistiu se na leitura dos resumos dos artigos e a segunda etapa realizou-se a seleção dos artigos que atendiam aos critérios de inclusão, e assim, efetuada a leitura integral dos mesmos.

Figura 1. Fluxograma de identificação, seleção, elegibilidade e inclusão dos estudos seguindo as recomendações PRISMA, Porto Alegre, RS, Brasil, 2021.

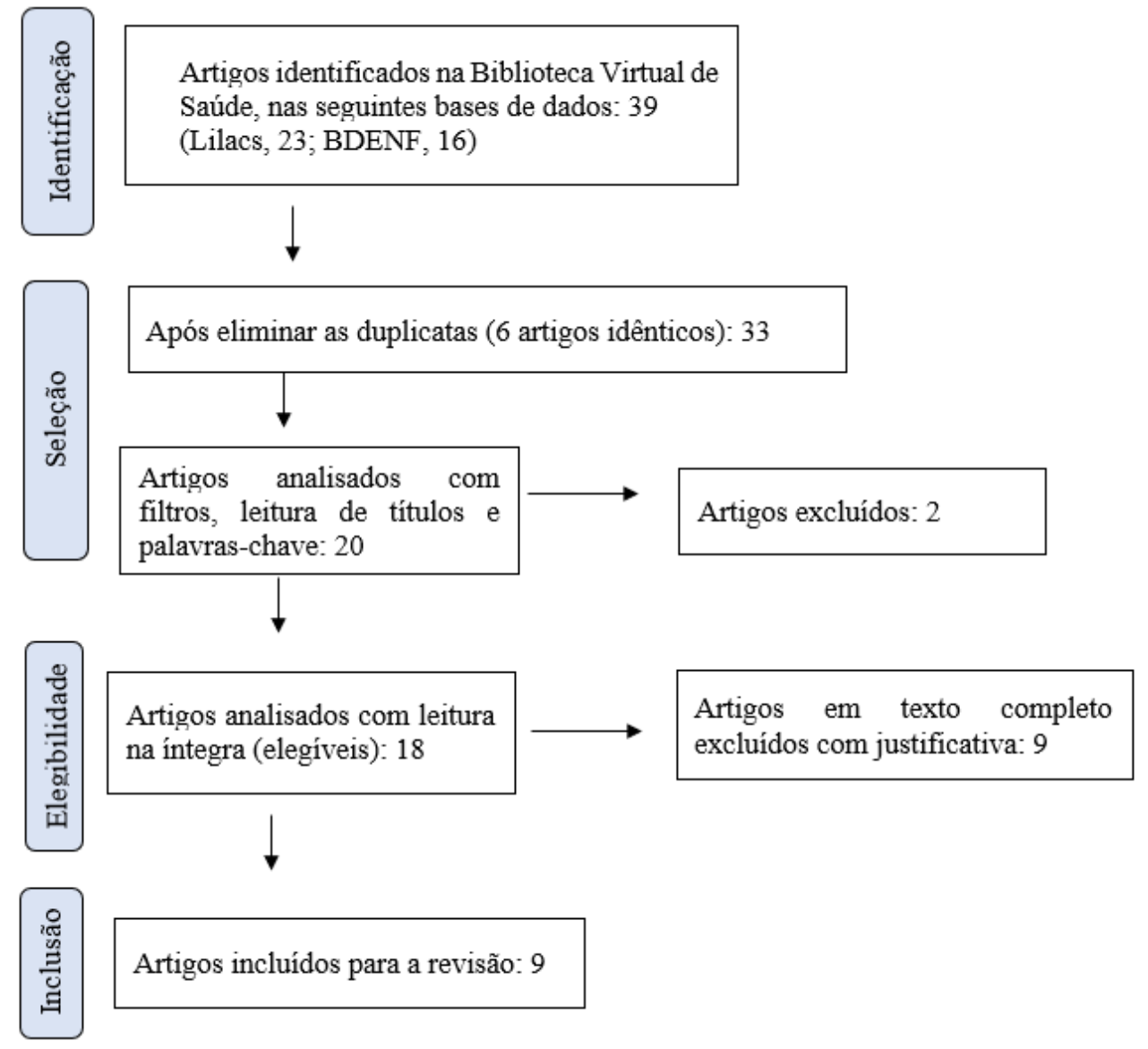

Fonte: Adaptado de Selçuk, A. A. (2019).

Em síntese, as estratégias de busca recuperaram 39 artigos. Durante o processo de seleção, foram excluídos 6 artigos duplicados e dois que não contemplavam os critérios de inclusão após a leitura do título, resumo e palavras-chave (primeira 
etapa). A leitura dos textos completos dos 18 artigos elegíveis (segunda etapa) resultou na exclusão de 9 (justificadas por se tratar de estudos versando sobre: educação em saúde de pacientes estomizados), restando 9 artigos. Para a análise e interpretação dos dados, realizou-se a síntese das informações extraídas dos artigos, utilizando um instrumento composto dos seguintes itens: título do artigo; autores e ano; periódicos; objetivo e resultados. Os dados extraídos e sintetizados são apresentados em quadro de caracterização da amostra

\section{Resultados e Discussão}

Foram selecionados 9 artigos que discutem as orientações de enfermagem aos pacientes ostomizados.

Quadro 1. Caracterização dos artigos selecionados para revisão integrativa, Porto Alegre, RS, Brasil, 2021.

\begin{tabular}{|c|c|c|c|c|}
\hline Título & Autores & Periódico & Objetivo & Resultados \\
\hline $\begin{array}{l}\text { Educação em saúde } \\
\text { com estomizados e } \\
\text { seus familiares: } \\
\text { possibilidade para } \\
\text { melhor qualidade de } \\
\text { vida }\end{array}$ & $\begin{array}{l}\text { Wild et al., } \\
2017\end{array}$ & $\begin{array}{l}\text { Revista de } \\
\text { Enfermagem } \\
\text { da UFSM }\end{array}$ & $\begin{array}{l}\text { Relatar a experiência de } \\
\text { enfermeiros frente à realização } \\
\text { de ações educativas com } \\
\text { pacientes estomizados e seus } \\
\text { familiares em uma Estratégia } \\
\text { Saúde da Família da Fronteira } \\
\text { Oeste do Rio Grande do Sul. }\end{array}$ & $\begin{array}{l}\text { No grupo educativo foram abordados } \\
\text { assuntos relativos à alimentação, sexualidade, } \\
\text { autocuidado e autoimagem, além dos } \\
\text { cuidados básicos com a estomia e manejo da } \\
\text { bolsa coletora. }\end{array}$ \\
\hline $\begin{array}{l}\text { Perfil de usuários e } \\
\text { motivos da consulta } \\
\text { de enfermagem em } \\
\text { estomaterapia }\end{array}$ & $\begin{array}{c}\text { Paczek et al., } \\
2020\end{array}$ & $\begin{array}{l}\text { Revista de } \\
\text { Enfermagem } \\
\text { da UFPE }\end{array}$ & $\begin{array}{l}\text { Analisar o perfil de usuários e } \\
\text { os motivos da consulta de } \\
\text { enfermagem em estomaterapia. }\end{array}$ & $\begin{array}{l}\text { Revela-se, quanto ao sexo, que não houve } \\
\text { diferença significativa; quanto à faixa etária, } \\
53,6 \% \text { dos participantes tinham } 65 \text { anos ou } \\
\text { mais de idade; a causa do estoma foi a doença } \\
\text { neoplásica maligna em } 65,5 \% \text { da amostra; o } \\
\text { motivo da consulta, em } 56 \% \text {, foi a troca de } \\
\text { bolsa de ostomia e } 50,4 \% \text { dos pacientes } \\
\text { realizaram somente uma consulta no período } \\
\text { analisado. }\end{array}$ \\
\hline $\begin{array}{l}\text { Efetividade da } \\
\text { educação a distância } \\
\text { no conhecimento de } \\
\text { enfermeiros sobre } \\
\text { estomias intestinais } \\
\text { de eliminação }\end{array}$ & $\begin{array}{l}\text { Alencar, } \\
2018\end{array}$ & $\begin{array}{c}\text { Revista } \\
\text { Gaúcha de } \\
\text { Enfermagem }\end{array}$ & $\begin{array}{l}\text { Avaliar a efetividade da } \\
\text { educação a distância no } \\
\text { conhecimento de enfermeiros da } \\
\text { atenção primária sobre estomias } \\
\text { intestinais de eliminação. }\end{array}$ & $\begin{array}{l}\text { A educação a distância pode ser uma } \\
\text { metodologia efetiva para educação } \\
\text { permanente de enfermeiros pois houve } \\
\text { melhora significativa nos conhecimentos dos } \\
\text { enfermeiros sobre a temática abordada. }\end{array}$ \\
\hline $\begin{array}{l}\text { Reabilitação de } \\
\text { pessoas com estomia } \\
\text { intestinal: revisão } \\
\text { integrativa }\end{array}$ & $\begin{array}{c}\text { Sasaki et al., } \\
2017\end{array}$ & $\begin{array}{l}\text { Revista de } \\
\text { Enfermagem } \\
\text { da UFPE }\end{array}$ & $\begin{array}{l}\text { Analisar a produção científica } \\
\text { nacional e internacional sobre a } \\
\text { reabilitação do estomizado } \\
\text { intestinal e as implicações para a } \\
\text { assistência. }\end{array}$ & $\begin{array}{l}\text { A reabilitação deve ser iniciada no hospital } \\
\text { com assistência especializada multidisciplinar } \\
\text { ao estomizado e sua família, ensino do } \\
\text { autocuidado com a estomia. }\end{array}$ \\
\hline $\begin{array}{l}\text { Avaliação do impacto } \\
\text { da capacitação no } \\
\text { trabalho para o } \\
\text { cuidado de pessoas } \\
\text { com Estomias }\end{array}$ & $\begin{array}{c}\text { Moraes et al., } \\
2019\end{array}$ & $\begin{array}{l}\text { Revista } \\
\text { Enfermagem } \\
\text { em Foco }\end{array}$ & $\begin{array}{l}\text { Avaliar o impacto da } \\
\text { capacitação de enfermeiros da } \\
\text { Atenção Primária da Saúde para } \\
\text { o cuidado à saúde das pessoas } \\
\text { com estomias. }\end{array}$ & $\begin{array}{l}\text { Demonstrou-se impacto positivo na } \\
\text { capacitação dos enfermeiros na Atenção } \\
\text { Primária à Saúde. }\end{array}$ \\
\hline $\begin{array}{l}\text { Análise de vídeos de } \\
\text { autocuidado no } \\
\text { YouTube sobre troca } \\
\text { de bolsas de estomias } \\
\text { intestinais }\end{array}$ & $\begin{array}{l}\text { Silva et al., } \\
\quad 2020\end{array}$ & Revista Rene & $\begin{array}{l}\text { Analisar os vídeos do YouTube } \\
\text { sobre o autocuidado durante a } \\
\text { troca das bolsas de estomia. }\end{array}$ & $\begin{array}{l}\text { Foram analisados } 32 \text { vídeos, no entanto, } \\
\text { identificou-se falhas no processo de educação } \\
\text { em saúde presente nesses vídeos. }\end{array}$ \\
\hline $\begin{array}{l}\text { O enfermeiro como } \\
\text { educador em saúde da } \\
\text { pessoa estomizada } \\
\text { com câncer Colorretal }\end{array}$ & $\begin{array}{l}\text { Farias et al., } \\
\quad 2019\end{array}$ & $\begin{array}{l}\text { Revista } \\
\text { Enfermagem } \\
\text { em Foco }\end{array}$ & $\begin{array}{l}\text { Conhecer a experiência de } \\
\text { enfermeiros no processo de } \\
\text { educação em saúde como } \\
\text { estratégia de ensino do } \\
\text { autocuidado a pessoa com } \\
\text { câncer com estomia intestinal. }\end{array}$ & $\begin{array}{l}\text { Emergiram três categorias temáticas do } \\
\text { estudo: o enfermeiro como educador, } \\
\text { incentivo ao autocuidado e atualização } \\
\text { profissional. Destacando-se o papel do } \\
\text { enfermeiro como educador. }\end{array}$ \\
\hline
\end{tabular}




\begin{tabular}{|c|c|c|c|c|}
\hline $\begin{array}{l}\text { Estratégias educativas } \\
\text { para pessoas com } \\
\text { estomia intestinal: } \\
\text { revisão integrativa }\end{array}$ & $\begin{array}{c}\text { Sousa et al., } \\
2017\end{array}$ & $\begin{array}{c}\text { Revista } \\
\text { Enfermagem } \\
\text { Atual In } \\
\text { Derme }\end{array}$ & $\begin{array}{l}\text { Analisar o conhecimento sobre } \\
\text { estratégias } \\
\text { empregadas pela enfermagem } \\
\text { para o ensino aprendizagem de } \\
\text { pessoas com estomia intestinal. }\end{array}$ & $\begin{array}{l}\text { Para que o processo de ensino aprendizagem } \\
\text { seja efetivo no campo da saúde, os recursos } \\
\text { didáticos empregados devem capacitar e } \\
\text { motivar os pacientes com vistas a } \\
\text { conseguirem incorporar novos significados, } \\
\text { propiciando mais autonomia. }\end{array}$ \\
\hline $\begin{array}{l}\text { Tecnologia para o } \\
\text { autocuidado da saúde } \\
\text { sexual e reprodutiva } \\
\text { de mulheres } \\
\text { estomizadas }\end{array}$ & $\begin{array}{l}\text { Albuquerque } \\
\text { et al., } 2016\end{array}$ & $\begin{array}{c}\text { Revista } \\
\text { Brasileira de } \\
\text { enfermagem } \\
\text { Reben }\end{array}$ & $\begin{array}{l}\text { validar uma tecnologia do tipo } \\
\text { cartilha impressa para o } \\
\text { autocuidado na saúde sexual e } \\
\text { reprodutiva de mulheres } \\
\text { estomizadas. }\end{array}$ & $\begin{array}{l}\text { A cartilha foi considerada válida segundo os } \\
\text { especialistas, com média global de CVI igual } \\
\text { a } 0,87 \text { e, segundo o público-alvo, com } 100 \% \\
\text { de concordância quanto à adequação da } \\
\text { organização, estilo da escrita, aparência e } \\
\text { motivação da cartilha. }\end{array}$ \\
\hline
\end{tabular}

Fonte: Autores.

Os estudos selecionados foram realizados no Brasil. Em relação ao ano de publicação, há predomínio de publicação do ano de 2017, com três artigos. O periódico Revista de Enfermagem da Universidade Federal de Pernambuco se destacou com a presença de dois artigos na amostra. Além disso, cabe salientar que a maioria dos trabalhos eram estudos qualitativos.

Os trabalhos foram realizados com profissionais de enfermagem, instrumentos educativos e usuários e suas interrelações com os cuidados com a estomia. O estudo apresenta a análise de artigos diversificados relacionados à temática orientações aos pacientes ostomizados. Para melhor discutir os estudos analisados, optou-se pela organização dos artigos agrupados em três categorias, conforme a temática dos estudos: Educação em saúde e a atuação do enfermeiro frente ao paciente ostomizado, abordagens de educação em saúde para promover o autocuidado e a reabilitação do paciente estomizado e estratégias e tecnologias para educação permanente das equipes sobre ostomias.

\section{Educação em saúde e a atuação do enfermeiro frente ao paciente estomizado}

Educação em saúde compreende ações e práticas que visam levar ao público em geral conhecimentos básicos a respeito de uma determinada condição de saúde, com a finalidade de capacitar esses indivíduos de forma que eles possam executar tarefas rotineiras da melhor maneira possível para promover a sua reabilitação ou a da pessoa sobre seus cuidados. Nesse sentido, o profissional enfermeiro, dentro da equipe de saúde assume um papel vital de propagação de conhecimentos adequados e fundamentados cientificamente, para melhorar a qualidade dos cuidados prestados no ambiente domiciliar, pelos cuidadores ou pelo próprio indivíduo (Wild et al., 2017).

Para Wild et al. (2017), a prática de educação em saúde com pacientes estomizados, vai muito além dos cuidados com o problema de saúde apresentado. Envolve fatores físicos, sociais, psicológicos e interferências nas rotinas diárias. O que vai exigir do paciente adaptações e processos que devem ser trabalhados pelos profissionais de saúde. Todo esse contexto biopsicossocial enfrentado, leva os autores a acreditar que o profissional deve adentrar nas mais variadas temáticas com os pacientes, desde os cuidados com higiene até mesmo questões de sexualidade. Segundo Wild et al (2017) ao efetivar práticas que possibilitem a interação entre profissionais e pacientes, espera-se estimular a autonomia, autoconhecimento, autocuidado, debate sobre valores, descobertas de sentimentos e amenizar tabus.

Wild et al. (2017) relata os desafios enfrentados pelos enfermeiros na assistência ao paciente estomizado corroborando com os achados dos perfis dos atendimentos dos enfermeiros estomaterapeutas em um serviço de estomaterapia de um centro de referência às pessoas com estomia do município de Porto Alegre, Rio Grande do Sul, em estudo publicado por Paczek et al. (2020). Os autores refletem sobre seus achados e encontram usuários com um perfil sensibilizado e emocionalmente impactado, pela estomia, devido a alteração na imagem corporal, podendo despertar sentimentos de vergonha, medo de rejeição social e incapacidade de autocuidado. O que demanda à equipe de enfermagem um atendimento altamente 
especializado ao portador de estomias. devendo o profissional enfermeiro ser capaz de gerenciar e planejar as ações de atenção a esse grupo específico (Wild et al., 2017; Paczek et al., 2020).

Alencar (2018) analisa uma outra face do processo de educação em saúde, onde é abordado os enfermeiros atuantes no acompanhamento e aconselhamento de pessoas com estomias. Foi observado que os mesmos quanto educadores em saúde ainda enfrentam o desafio de deixar de lado a abordagem técnica e mecanicista frente ao paciente que são na sua maioria leigos na temática. E as dificuldades enfrentadas para migrar para uma metodologia holística, integral e multidisciplinar de acolhimento e atendimento do paciente estomizado.

\section{Abordagens de educação em saúde para promover o autocuidado e a reabilitação do paciente estomizado}

Sasaki et al (2017) relata que as estratégias educativas dentro desse contexto devem visar a autonomia, o autocuidado e a reabilitação do paciente nessa condição de saúde. Permitindo que o mesmo se sinta confortável e autônomo perante suas atividades cotidianas e melhorando a percepção da sua autoimagem. Sousa et al (2017), relata que as abordagens dialógicas e tecnológicas são as mais praticadas sendo muitas vezes complementares uma da outra. Se por um lado os desafios da prática de educação dialógica é a comunicação eficiente e a tradução do conteúdo técnico científico, por outro a prática que envolve tecnologias se depara com as desigualdades de acessos e limitações de conhecimentos para manusear aparelhos, mídias e aplicativos por uma parcela da população. Sendo necessário adaptações no processo de ensino aprendizagem para essas estratégias (Sasaki et al., 2017; Moraes et al., 2019).

Dentro das tecnologias educativas para a promoção do autocuidado, existe uma escassez dentro de algumas redes sociais de conteúdos próprios e adequadamente apresentados para esses pacientes, como no Youtube, por exemplo. Os achados de um estudo demonstram que a maioria dos vídeos contidos na plataforma são feitos por pacientes com estomias e apresentam algum tipo de erro ou prática inadequada. Apesar da aceitação para se expor dessa maneira ser notável e o alcance da mídia ser maior do que de um grupo dentro de uma unidade de saúde ou comunidade, essas técnicas abordadas incorretamente podem ser disseminadas e comprometer o processo de autocuidado de outros indivíduos estomizados (Silva et al., 2020).

De encontro a isso, se faz necessário à validação do processo e do conteúdo a ser propagado nas redes sociais e nas mais diversas tecnologias a serem utilizadas na educação em saúde sobre estomias, a fim de permitir que os pacientes possam se beneficiar do que estiverem assistindo ou lendo. $\mathrm{O}$ uso de tecnologias educativas validades atribui maior qualidade ao conteúdo produzido no processo de ensino-aprendizagem. (Moraes et al., 2019; Farias; Nery; Santana, 2019).

\section{Estratégias e tecnologias para educação permanente das equipes sobre estomias}

A educação permanente em saúde consiste na prática de ensino e treinamento contínuos das equipes de trabalho, com o objetivo de melhorar a qualidade assistencial prestado aos pacientes. Sendo uma prática rotineira nos estabelecimentos de saúde. A literatura nos traz as capacitações como estratégia mais comum e de fácil aplicabilidade nos mais diversos ambientes e realidades. Com tudo se diferenciam as tecnologias e metodologias aplicadas para a construção do conhecimento em foco (Sousa et al., 2017; Albuquerque et al., 2016).

Corroboram-se entre si, os autores, Alencar (2018) e Sousa et al. (2017), pois afirmam que no cuidado ao paciente estomizado, surge a problemática da necessidade de conhecimentos específicos por parte dos enfermeiros que prestam assistência a esses pacientes. Conhecimentos que não são aprofundados na graduação, sendo, portanto, necessário o profissional realizar atualização científica sobre a temática. Na atenção primária à saúde, as noções básicas de cuidados de estomaterapia se fazem necessários para a disseminação do conhecimento na equipe de saúde, pois trata-se de um ambiente de cuidado no qual enfermeiros e a equipe assistencial estarão rotineiramente cuidando de usuários portadores de estomas. 
Nesse cenário, a avaliação do impacto das capacitações sobre os profissionais enfermeiros e sobre as equipes. Por meios de ferramentas de feedback e debriefing são eficientes no contexto de educação permanente sobre pacientes estomizados (Moraes et al., 2019). Outra metodologia de educação oportuna para o cenário atual, é de educação à distância (EaD). Dados significativos da construção desse conhecimento se mostram promissores e com práticas viáveis com tecnologias e abordagens cada dia mais dinâmicas (Sousa et al, 2017).

Todo o processo de educação em saúde, incluindo as relacionadas a ostomias intestinais, dentro de instituições públicas ou privadas de saúde que se relacionam às práticas dos profissionais de enfermagem, necessita do acompanhamento de um profissional enfermeiro qualificado para assegurar a qualidade da assistência prestada e a segurança do paciente (Moraes et al., 2019).

\section{Conclusão}

As orientações em saúde aos pacientes estomizados envolvem um perfil de paciente extremamente sensibilizado emocionalmente devido a sua nova forma corporal, nessa situação, a atuação do enfermeiro frente ao paciente com essa condição de saúde é uma tarefa que deve envolver uma metodologia holística, integral e multidisciplinar de acolhimento no atendimento desse paciente.

Referente às estratégias educativas conclui-se que as abordagens dialogadas e tecnológicas são as mais praticadas, sendo muitas vezes complementares, porém, ainda se observa barreiras na comunicação eficiente entre pacientes e profissionais de enfermagem devido ao uso de uma linguagem técnica pelos profissionais orientadores. No entanto, cabe salientar que há escassez de estudos voltados a temática, sendo necessário a realização de trabalhos na área de enfermagem.

Portanto, se faz necessário a implementação de políticas públicas voltadas a disseminação do conhecimento para o autocuidado dos indivíduos portadores de estomas, bem como estratégias de educação permanente aos profissionais de saúde.

\section{Referências}

Albuquerque, A. F. L. L., Pinheiro, A. K. B., Linhares, F. M. P., \& Guedes, T. G. (2016). Tecnologia para o autocuidado da saúde sexual e reprodutiva de mulheres estomizadas. Revista Brasileira de Enfermagem, 69(6), 1164-1171.

Alencar, D. D. C., Andrade, E. M. L. R., Rabeh, S. A. N., \& Araújo, T. M. E. D. (2018). Efetividade da educação a distância no conhecimento de enfermeiros sobre estomias intestinais de eliminação. Revista Gaúcha de Enfermagem, 39.

Andrade, R. S. D., Martins, J. M., Medeiros, L. P. D., Souza, A. J. G. D., Torres, G. D. V., \& Costa, I. K. F. (2017). Aspectos sociodemográficos, clínicos e de autocuidado de pessoas com estomas intestinais. Rev. enferm. UERJ, e19368-e19368.

Brasil. (2019). Guia de atenção à saúde da pessoa com estomia. Brasília, DF: Ministério da Saúde.

Caetano, C. M., Beuter, M., da Silva Jacobi, C., Mistura, C., da Costa Rosa, B. V., \& Seiffert, M. A. (2014). O cuidado à saúde de indivíduos com estomias. Revista de Atenção à Saúde, 12(39), 59-65.

De Farias, D. L. S., Nery, R. N. B., \& de Santana, M. E. (2019). O enfermeiro como educador em saúde da pessoa estomizada com câncer colorretal. Enferm. foco (Brasília), 10(1), 35-39

De Morais, F. F., Santos, J. D. M., de Vera, S. O., Oliveira, R. G. A., Andrade, E. M. L. R., \& Araújo, S. N. M. (2018). Manejo de estomas intestinais de eliminação: conhecimento do cuidador. O Mundo da Saúde, 42(4), 823-844.

De Sousa, A. R. A., de Menezes, L. C. G., Miranda, S. M., \& Cavalcante, T. B. (2017). Estratégias educativas para pessoas com estomia intestinal: revisão integrativa. Revista Enfermagem Atual In Derme, 81(19).

Dos Santo, J. C., Donoso, M. T. V., da Silveira Sete, A., Lima, L. K. B., de Matos, S. S., \& de Aguilar, I. M. (2020). Characterization of people with intestinal stomas hospitalized in private hospital/Caracterização de pessoas com estomas intestinais internadas em hospital privado/Caracterización de personas con estomas intestinales internadas en hospital privado. Revista de Enfermagem da UFPI, 9(1).

Martins, P. A. D. F., \& Alvim, N. A. T. (2011). Perspectiva educativa do cuidado de enfermagem sobre a manutenção da estomia de eliminação. Revista Brasileira de Enfermagem, 64(2), 322-327.

Mendes, K. D. S., Silveira, R. C. C. P., \& Galvão, C. M. (2008). Integrative literature review: a research method to incorporate evidence in health care and nursing. Texto contexto enferm, 17(4), 758-64. 
Research, Society and Development, v. 10, n. 9, e31310918086, 2021

(CC BY 4.0) | ISSN 2525-3409 | DOI: http://dx.doi.org/10.33448/rsd-v10i9.18086

Moraes, J. T., Silva, A. E., Gontijo, T. L., Ribeiro, R. F., \& Faria, R. D. G. S. (2019). Avaliação do impacto da capacitação no trabalho para o cuidado de pessoas com estomias. Enferm. foco (Brasília), 93-98.

Paczek, R. S., Engelmann, A. I., Perini, G. P., Aguiar, G. P. S. D., \& Duarte, E. R. M. (2020). Perfil de usuários e motivos da consulta de enfermagem em estomaterapia. Rev. enferm. UFPE on line, 1-7.

Sasaki, V. D. M., Teles, A. A. D. S., Lima, M. S. D., Barbosa, J. C. C., Lisboa, B. B., \& Sonobe, H. M. (2017). Reabilitação de pessoas com estomia intestinal: Revisão integrativa. Rev. enferm. UFPE on line, 1745-1754.

Selçuk, A. A. (2019). A guide for systematic reviews: PRISMA. Turkish archives of otorhinolaryngology, 57(1), 57.

Silva, A. L., \& Shimizu, H. E. (2006). O significado da mudança no modo de vida da pessoa com estomia intestinal definitiva. Revista Latino-americana de enfermagem, 14(4), 483-490.

Silva, B. W. A. C. D., Araújo, A. K. D., Medeiros, M. B. C., Melo, V. L. D., Sena, J. F. D., \& Costa, I. K. F. (2020). Análise de vídeos de autocuidado no YouTube sobre troca de bolsas de estomias intestinais. Rev. Rene. 21:e44275.

Silva, C. R. D. T., Andrade, E. M. L. R., Luz, M. H. B. A., Andrade, J. X., \& Silva, G. R. F. D. (2017). Qualidade de vida de pessoas com estomias intestinais de eliminação. Acta Paulista de Enfermagem, 30(2), 144-151.

Vidal, S. A., Samico, I. C., Frias, P. G. D., \& Hartz, Z. M. D. A. (2011). Estudo exploratório de custos e conseqüências do pré-natal no Programa Saúde da Família. Revista de Saúde Pública, 45, 467-474.

Wild, C. F., Favero, N. B., Salbego, C., Vale, M. G. D., Silva, J. D. R. P. D., \& Ramos, T. K. (2016). Educação em saúde com estomizados e seus familiares: possibilidade para melhor qualidade de vida. Rev. enferm. UFSM, 290-297. 